\title{
AOR
}

Selected Papers of \#AolR2020:

The $21^{\text {st }}$ Annual Conference of the Association of Internet Researchers

Virtual Event / 27-31 October 2020

\section{INVESTIGATING BOTS AND COORDINATED INFLUENCE CAMPAIGNS IN TWITTER DISCUSSIONS OF THE 2019-20 IRAN PROTESTS}

Ehsan Dehghan

Digital Media Research Centre, Queensland University of Technology

Brenda Moon

Digital Media Research Centre, Queensland University of Technology

Tobias Keller

Digital Media Research Centre, Queensland University of Technology

Timothy Graham

Digital Media Research Centre, Queensland University of Technology

Axel Bruns

Digital Media Research Centre, Queensland University of Technology

Daniel Angus

Digital Media Research Centre, Queensland University of Technology

\section{Introduction}

Twitter is a vital platform for organizing, coordinating, and amplifying voices during protests all around the world, especially in non-democratic countries (Tufekci 2017). Yet, not only does this platform allow minorities and protesters to have a voice and be heard, but also to spread propaganda, sow discord, and crowd out genuine voices sometimes even through the use of social bots (Shao et al. 2018).

Although Twitter is a globally used platform for protest movements, many studies focus on English-speaking and democratic countries representing the so-called West. Only a very few studies focus on the Middle East and, if so, limit their investigation to the Suggested Citation (APA): Dehghan, Ehsan. Moon, Brenda. Keller, Tobias. Graham, Timothy. Bruns, Axel, and Angus, Daniel. (2020, October). Investigating bots and coordinated influence campaigns in Twitter discussions of the 2019-20 Iran protests. Paper presented at AoIR 2020: The $21^{\text {th }}$ Annual Conference of the Association of Internet Researchers. Virtual Event: AolR. Retrieved from http://spir.aoir.org. 
English content (e.g. Featherman 2015; Thieltges et al. 2018). We overcome this research gap and investigate the Persian-, English-, and Arabic- speaking actors and content during Twitter discussions of the 2019-20 protests in Iran.

In November 2019, a sudden increase in fuel prices triggered a series of protests spreading to 21 Iranian cities. Although the protests started in reaction to the price hike, demands and slogans soon took an anti-regime turn, and some protests led to violent riots. The Internet was shut down for over 100 hours, and different sources estimate the number of fatalities between 300 (Amnesty International 2019) and 1500 (Reuters 2019).

This work-in-progress paper examines the first-hand accounts and wider perspectives involved in the discussions of the protests on Twitter. The particularities of the Iranian context both online and offline make this a significant and timely investigation into the interrelationship of social media and democracy in this unique context.

Our study investigates the composition, themes, and discourses involved in the complex domestic and international tensions in the Twitter discussion of the 2019-20 protests in Iran. Given the increasing debate and attention to misinformation campaigns and the increasing identification of bots originating from Iran and Saudi Arabia by social media platforms (France-Presse 2019; Owen Jones 2020; Roth 2019), we first map the Twittersphere revolving around the Iran protests and detect key clusters (RQ1), and, second, focus on the evidence of the presence of bots, bot networks, and coordinated misinformation campaigns and political astroturfing (Keller et al. 2019) in these clusters (RQ2).

\section{Method}

We collected tweets discussing Iran protests using a range of keywords and hashtags, in Persian, English, and Arabic. In total, 5,560,247 tweets were collected from 757,927 accounts, between 13/11/2019 and 19/01/2020.

We relied on Botometer to detect social bots within this dataset (e.g. Varol et al. 2017). Botometer is a machine learning algorithm that detects accounts that behave or look similar to known suspicious accounts (Davis et al. 2016; Yang et al. 2019). The tool has been used to study political discussions from a variety of global contexts (e.g.

Broniatowski et al. 2018; Keller and Klinger 2019; Shao et al. 2018). We manually reviewed a subset of the account classifications to ensure the accuracy of Botometer in our context.

To answer RQ1, we visualised the retweet network. We then identified the largest 20 clusters and qualitatively investigated the Twitter profiles of the top users (highest degree) in each cluster to identify its political position. To answer RQ2, we focus on the bot scores from Botometer to identify those clusters in the retweet network that show suspiciously high levels of automated accounts.

\section{Findings}


Our investigation of the retweet network (Figure 1) indicates strong homophilous networks of users formed around ideological, political, and discursive interests. These clusters in the network reproduce the linguistic, regional, and political affiliations of users discussing Iran protests. For instance, there are distinct clusters formed around retweets by pro-regime accounts, anti-regime groups such as the supporters of the Pahlavi dynasty, the militant anti-regime organisation MEK, reformists, journalists and news organisations, and users from various Arabic-speaking countries.

Figure 1: Retweet network showing distinct clustering tendencies

Our preliminary results indicate a strong presence of bots in two of the largest clusters (Figure 2). Botometer assigns a 'botness score' between 0 (human) and 1 (bot) to each account it assesses. While most ordinary accounts received botness scores of 0.3 or below, Figure 2 shows that two clusters in the network contain an unusually high proportion of accounts with scores of 0.5 or higher. Interestingly, these clusters reflect antagonistic political affiliations-one consists mainly of pro-regime Iranian accounts $(23,755$ accounts $)$, the other of anti-regime accounts tweeting in Arabic $(14,052$ accounts). 


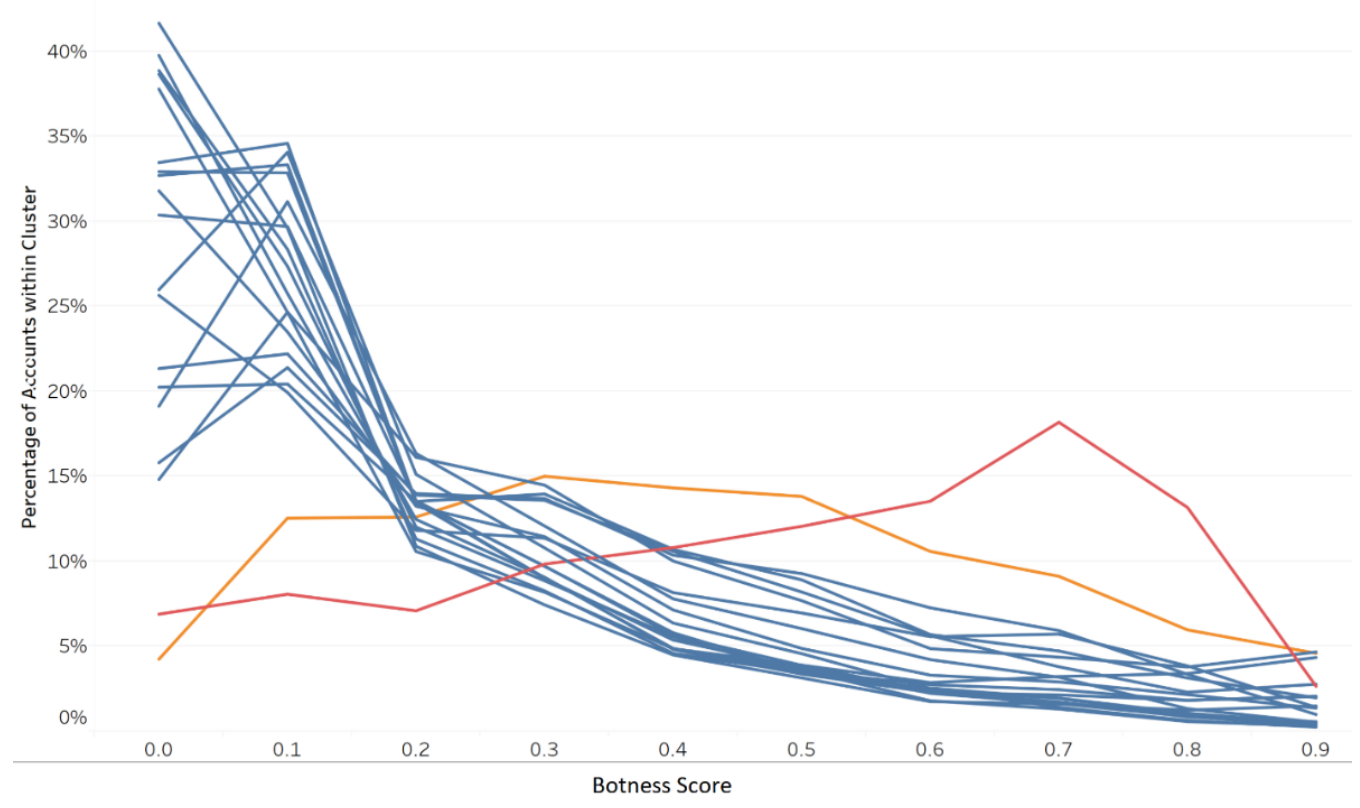

Figure 2: Botness scores for the 20 largest clusters. An Arabic-tweeting cluster (red) and a pro-Iranian regime cluster (orange) show suspiciously high botness scores

\section{Discussion and Future Steps}

The next steps in this study will be to extend this analysis to the networks of @mentions and @replies, each of which reveals different dynamics. We will also use computational discourse analysis to examine the topics and themes discussed by each of the major clusters in the network, to investigate the dynamics of this complex interaction environment.

This study enhances our knowledge of the Persian, Arabic, and English-speaking actors and communities taking part in the public debate on Iran protests. Preliminary results indicate the presence of several communities, each of which represents the discussion from its own discursive positions. Two of the clusters-interestingly each with hyperpartisan political positions-show a high level of automated activity. Such results have direct implications for democratic projects in the region, showing the presence and spread of state-sponsored coordinated activities online. The broader findings from this study will therefore also be valuable for understanding digital activism, protests, and political communication in authoritarian and semi-authoritarian contexts.

\section{References}

Amnesty International. 2019. "Iran's Authorities Carrying out Vicious Post-Protest Crackdown." Amnesty International. Retrieved February 27, 2020 (https://www.amnesty.org/en/latest/news/2019/12/iran-thousands-arbitrarilydetained-and-at-risk-of-torture-in-chilling-post-protest-crackdown/).

Broniatowski, David A., Amelia M. Jamison, SiHua Qi, Lulwah AlKulaib, Tao Chen, Adrian Benton, Sandra C. Quinn, and Mark Dredze. 2018. "Weaponized Health 
Communication: Twitter Bots and Russian Trolls Amplify the Vaccine Debate." American Journal of Public Health 108(10):1378-84.

Davis, Clayton A., Onur Varol, Emilio Ferrara, Alessandro Flammini, and Filippo Menczer. 2016. "BotOrNot: A System to Evaluate Social Bots." Proceedings of the 25th International Conference Companion on World Wide Web - WWW'16 Companion 273-74.

Featherman, Chris. 2015. Discourses of Ideology and Identity: Social Media and the Iranian Election Protests. New York; London: Routledge, Taylor \& Francis Group.

France-Presse, Agence. 2019. "Twitter Blocks Accounts Linked to Saudi 'state-Backed' Manipulation Effort." The Guardian, December 20.

Keller, Franziska B., David Schoch, Sebastian Stier, and JungHwan Yang. 2019. "Political Astroturfing on Twitter: How to Coordinate a Disinformation Campaign." Political Communication 1-25.

Keller, Tobias R., and Ulrike Klinger. 2019. "Social Bots in Election Campaigns: Theoretical, Empirical, and Methodological Implications." Political Communication 36(1):171-89.

Owen Jones, Marc. 2020. "Saudi Arabia's Bot Army Flourishes as Twitter Fails to Tame the Beast." Middle East Eye. Retrieved February 27, 2020 (http://www.middleeasteye.net/opinion/despite-twitter-culls-riyadhsdisinformation-network-still-going-strong).

Reuters. 2019. "Special Report: Iran's Leader Ordered Crackdown on Unrest - 'Do Whatever It Takes to End It." Reuters, December 23.

Roth, Yoel. 2019. "Information Operations on Twitter: Principles, Process, and Disclosure." Retrieved February 27, 2020 (https://blog.twitter.com/en_us/topics/company/2019/information-ops-ontwitter.html).

Shao, Chengcheng, Giovanni Luca Ciampaglia, Onur Varol, Kai-Cheng Yang, Alessandro Flammini, and Filippo Menczer. 2018. "The Spread of Low-Credibility Content by Social Bots." Nature Communications 9(1):4787.

Thieltges, Andree, Orestis Papakyriakopoulos, Juan Carlos Medina Serrano, and Simon Hegelich. 2018. "Effects of Social Bots in the Iran-Debate on Twitter." ArXiv:1805.10105 [Cs].

Tufekci, Zeynep. 2017. Twitter and Tear Gas: The Power and Fragility of Networked Protest. New Haven; London: Yale University Press. 
Varol, Onur, Emilio Ferrara, Clayton A. Davis, Filippo Menczer, and Alessandro Flammini. 2017. "Online Human-Bot Interactions: Detection, Estimation, and Characterization." ArXiv:1703.03107 [Cs].

Yang, Kai-Cheng, Onur Varol, Clayton A. Davis, Emilio Ferrara, Alessandro Flammini, and Filippo Menczer. 2019. "Arming the Public with Artificial Intelligence to Counter Social Bots." Human Behavior and Emerging Technologies 1(1):48-61. 